\title{
Two-gap to single-gap superconducting transition on a honeycomb lattice in $\mathrm{Ca}_{1-x} \mathrm{Sr}_{x} \mathrm{AlSi}$
}

\author{
Dorota I. Walicka $\odot,{ }^{1}$ Zurab Guguchia,${ }^{2}$ Jorge Lago,${ }^{1,3}$ Olivier Blacque, ${ }^{1}$ KeYuan Ma, ${ }^{1}$ Huanlong Liu, ${ }^{4}$ \\ Rustem Khasanov (i), ${ }^{2}$ and Fabian O. von Rohr (1, $^{1, *}$ \\ ${ }^{1}$ Department of Chemistry, University of Zurich, CH-8057 Zurich, Switzerland \\ ${ }^{2}$ Laboratory for Muon Spin Spectroscopy, Paul Scherrer Institute, CH-5232 Villigen, Switzerland \\ ${ }^{3}$ Departamento de Química Inorgánica, Universidad del País Vasco, UVP/EHU, E-48080 Bilbao, Spain \\ ${ }^{4}$ Department of Physics, University of Zurich, $\mathrm{CH}-8057$ Zurich, Switzerland
}

(Received 3 July 2020; revised 14 June 2021; accepted 16 June 2021; published 26 August 2021)

\begin{abstract}
We report on the structural and microscopic superconducting properties of the $\mathrm{Ca}_{1-x} \mathrm{Sr}_{x} \mathrm{AlSi}$ solid solution. Specifically, we have realized the continuous solid solution, which for all members, other than $x=0(\mathrm{CaAlSi})$, crystallizes in the $\mathrm{AlB}_{2}$-type structure. For CaAlSi, we present an improved structural model where all $\mathrm{Al} / \mathrm{Si}$ layers are buckled, leading to a 6-folded structure along the crystallographic $c$ direction. We, furthermore, find indications for the structural instability in the parent compound CaAlSi to enhance the superconductivity across the solid solution. Our investigation of the magnetic penetration depths by means of muon-spin rotation experiments reveals that CaAlSi is a two-gap superconductor, that SrAlSi is a single-gap superconductor, and that there is a continuous transition from one electronic state to the other across the solid solution. Hence, we show that the $\mathrm{Ca}_{1-x} \mathrm{Sr}_{x} \mathrm{AlSi}$ solid solution is a highly tunable two-gap to single-gap superconducting system on a honeycomb lattice, where the superconductivity is strongly connected to a structural instability, i.e., the buckling of the $\mathrm{Al} / \mathrm{Si}$ layers.
\end{abstract}

DOI: 10.1103/PhysRevResearch.3.033192

\section{INTRODUCTION}

The increase of the superconducting transition temperature at a structural phase boundary is a widely observed behavior in various superconductors [1-4]. The relationships between structural instabilities and superconductivity has been a recurring theme in superconductivity research. These structural phase transitions are associated with a phonon softening, which influences the phonon-mediated Cooper pairing and henceforth may lead to a enhancement of the superconductivity $[5,6]$. In particular, many of the known superconductors are chemically and electronically located close to structural phase instabilities, e.g., $\mathrm{Nb}_{3} \mathrm{Sn}$-related, bismuth-oxide, or graphite intercalated superconductors [7-9].

The recent discovery of superconductivity in magic-angle bilayer graphene has reignited the interest in superconductors with honeycomb lattices [10]. The most prominent bulk superconductor with (boron) honeycomb layers sandwiched between $\mathrm{Mg}$ (II) layers, is $\mathrm{MgB}_{2}$ with a critical temperature of $T_{\mathrm{c}}=39 \mathrm{~K}$ [11]. This remains the highest critical temperature at ambient pressure to-date, among materials, in which Cooper pairing is believed to be mediated by phonons, and described by the BCS (Bardeen-Cooper-Schrieffer) theory [12].

\footnotetext{
*fabian.vonrohr@uzh.ch

Published by the American Physical Society under the terms of the Creative Commons Attribution 4.0 International license. Further distribution of this work must maintain attribution to the author(s) and the published article's title, journal citation, and DOI.
}

The superconducting properties of $\mathrm{MgB}_{2}$ are caused by two types of electrons at the Fermi level with widely differing behaviors leading to the opening of two superconducting gaps $[13,14]$.

Other honeycomb bulk superconductors are intercalated variants of graphite. Prominent examples of this group are $\mathrm{CaC}_{6}$ with a critical temperature of $T_{\mathrm{c}}=11 \mathrm{~K}$, which increases up to $15.1 \mathrm{~K}$ at a pressure of $p=8 \mathrm{GPa}$ [9] and $\mathrm{YbC}_{6}$ with a critical temperature of $T_{\mathrm{c}}=6.5 \mathrm{~K}$ [15]. Here the superconductivity is likely caused by intercalant phonons $[16,17]$ or by an enhanced electron-phonon coupling through acoustic plasmons [18].

Besides these binary superconductors, there are only few ternary honeycomb-based superconductors known. Recently, SrPtAs with a critical temperature of $T_{\mathrm{c}} \approx 2.4 \mathrm{~K}$ has been of great interest, since it was predicted to be an intrinsic topological superconductor with a $d+i d$ gap on a layered hexagonal lattice [19-21]. Among the ternary honeycomb-based superconductors has the MAlSi ( $\mathrm{M}=\mathrm{Ca}, \mathrm{Sr}, \mathrm{Ba})$ family been most prominently discussed, with their critical temperatures of up to $T_{\mathrm{c}}=7.8 \mathrm{~K}$ [22-30]. SrAlSi and BaAlSi crystallize in the $\mathrm{AlB}_{2}$-type structure with $\mathrm{Al} / \mathrm{Si}$ honeycomb layers, which are intercalated by the earth-alkaline metal $[25,26,30]$. The structure of $\mathrm{CaAlSi}$ is related to the $\mathrm{AlB}_{2}$ structure, but it is more complex. It is believed that this material can crystallize in few polymorphic structures: denoted as $1 \mathrm{H}, 5 \mathrm{H}$, and $6 \mathrm{H}$ structures, here $\mathrm{H}$ stands for hexagonal and the number represents the amount of honeycomb Al/Si layers in the unit cell [31]. The critical temperatures vary between the different polymorphs between $T_{\mathrm{c}}=5.7$ to $7.8 \mathrm{~K}$, however, all of them were reported to consist partly of planar honeycomb $\mathrm{Al} / \mathrm{Si}$ layers $[32,33]$. 
$\mathrm{CaAlSi}$ and SrAlSi superconductors are widely believed to be conventional phonon-mediated superconductors. There are, however, also reports that indicate uncommon superconducting properties, such as, e.g., unconventional pressure effects $[25,34]$ or field-dependent muon spin rotation $(\mu \mathrm{SR})$ measurements on CaAlSi [35]. Especially noteworthy are a series of detailed specific heat capacity measurements, which have indicated different mechanisms behind the superconductivity in CaAlSi and SrAlSi [25]. Lorenz et al. suggested that even though these two compounds are isoelectronic and belong to the same structural family their properties vary significantly: SrAlSi can be best described by a weak coupling BCS theory and has negative response on applied pressure, while CaAlSi was found to be best described by a strong coupling BCS theory and has a positive pressure coefficient in the low-pressure $(p<1.2 \mathrm{GPa})$ regime. Moreover, both of them were predicted to be one gap superconductors in contrary to $\mathrm{MgB}_{2}[36,37]$.

Here, we investigate the structural and the microscopic superconducting properties of the $\mathrm{Ca}_{1-x} \mathrm{Sr}_{x} \mathrm{AlSi}$ solid solution. Specifically, we have realized the continuous solid solution, which for all members, other than $x=0$, crystallizes in the $\mathrm{AlB}_{2}$-type structure. We present an improved structural model for the parent compound CaAlSi by means of single-crystal $\mathrm{X}$-ray diffraction. We find that all honeycomb-type A/Si layers of CaAlSi are slightly buckled, and none of them are planar as in the prototypical $\mathrm{AlB}_{2}$ structure. Our results indicate that the structural instability in CaAlSi enhances the superconductivity across the solid solution. This is further supported by an investigation of the London magnetic penetration depths $\lambda^{-2}$, where we find strong evidence for the transition of a twogap $s+s$-wave superconducting gap to a single-gap $s$-wave model, which we find to coincide with the disappearance of the structural distortion across the solid solution.

\section{METHODS}

Synthesis-All compounds were synthesized from the pure elements (Ca 99.99\% Aldrich; Sr 99.99\% Aldrich; Al 99.9995\% Acros Organic; Si 99.95\% Aldrich). Ca and Sr were handled inside an argon glovebox to prevent the oxidation of the reactants. Stoichiometric amounts of the metals were arc-melted in an argon atmosphere on a water-cooled copper plate (anode) with the use of a tungsten tip (cathode). A $\mathrm{Zr}$ sponge was coheated in the system to further purify the reaction atmosphere from oxygen or water. In case of SrAlSi, a $5 \%$ excess of strontium was used for the highest purity product. The metals were melted together and remelted three times, each time flipping the sample over, in order to provide a maximized homogeneity of the prepared sample. Single crystals of CaAlSi, $\mathrm{Ca}_{0.8} \mathrm{Sr}_{0.2} \mathrm{AlSi}, \mathrm{Ca}_{0.6} \mathrm{Sr}_{0.4} \mathrm{AlSi}, \mathrm{Ca}_{0.5} \mathrm{Sr}_{0.5} \mathrm{AlSi}$, and SrAlSi were prepared by slowly decreasing the applied voltage over a few seconds.

Diffraction-Powder x-ray diffraction (PXRD) patterns were obtained on a STOE STADIP diffractometer equipped with a Ge-monochromator using $\mathrm{Cu}-\mathrm{K}_{\alpha 1}$ radiation $(\lambda=$ $1.54051 \AA$ ). All patterns were measured in the $5-90^{\circ} 2 \Theta$ range with a scan step of $0.015^{\circ}$. The cell parameters were obtained by performing LeBail fits using the FULLPROF program package [38]. Single crystal x-ray diffraction
(SXRD) data were collected on a Rigaku OD Synergy (Pilatus 200K Hybrid Pixel Array detector) diffractometer for single crystal from nominal compositions $\mathrm{SrAlSi}, \mathrm{Ca}_{0.8} \mathrm{Sr}_{0.2} \mathrm{AlSi}$, $\mathrm{Ca}_{0.6} \mathrm{Sr}_{0.4} \mathrm{AlSi}, \mathrm{Ca}_{0.5} \mathrm{Sr}_{0.5} \mathrm{AlSi}$, and on a Rigaku OD SuperNova (Atlas CCD detector) diffractometer for CaAlSi, both equipped with an Oxford liquid-nitrogen cryostream cooler. A single wavelength $\mathrm{x}$-ray source from a micro-focus sealed $\mathrm{X}$-ray tube was used for the analyses $\left(\mathrm{Cu}-\mathrm{K}_{\alpha}\right.$ radiation, $\lambda=$ $1.54184 \AA$ ). Suitable single crystals were manipulated into polybutene oil, mounted on a flexible loop fixed on a goniometer head and transferred to the diffractometer. Data collections, data reductions, and analytical absorption corrections [39] were performed with the program suite CrysAlisPro. Using Olex2 [40], the structures were solved with the SHELXT small-molecule structure solution program [41] and refined with the SHELXL program package [42] by full-matrix least-squares minimization on $\mathrm{F}^{2}$. Elemental analysis was performed with Zeiss GeminiSEM 450 field emission scanning electron microscope equipped with X-ray detector X-MAX80, AZTec Advanced, Oxford. Ten spectra were acquired for every sample, with applied voltage of $20 \mathrm{kV}$ and at $12 \mathrm{~mm}$ working distance.

Physical Properties-Magnetic measurements were carried out on a Quantum Design Magnetic Properties Measurement System (MPMS XL) equipped with a reciprocating sample option (RSO) and a $7 \mathrm{~T}$ magnet. The specific heat capacity was measured with the specific heat capacity option of the Physical Property Measurement System (PPMS, Quantum Design Inc.) in the temperature range between 2 and $16 \mathrm{~K}$ ( $2 \mathrm{~K}$ to $10 \mathrm{~K}$ for CaAlSi) in the field of $0 \mathrm{~T}$ and $3 \mathrm{~T}$. The $\mu \mathrm{SR}$ experiments were carried out at the Swiss Muon Source $(\mathrm{S} \mu \mathrm{S})$ Paul Scherrer Insitute, Villigen, Switzerland using the low-background GPS ( $\pi$ M3 beamline) instrument, the highfield HAL-9500 $\mu$ SR spectrometer ( $\pi \mathrm{E} 3$ beamline), equipped with BlueFors vacuum-loaded cryogen-free dilution refrigerator (DR) and high-pressure GPD instrument ( $\mu \mathrm{E} 1$ beamline), equipped with Oxford Instruments Heliox ${ }^{3} \mathrm{He}$ cryostat. For the experiment on the HAL-9500 instrument, a pellet with a diameter of $10 \mathrm{~mm}$ was used. For pressure experiments, three pellets of $5.9 \mathrm{~mm}$ in diameter were used. Pressures up to 1.9 GPa were generated in a double wall piston-cylinder type of cell made of MP35N material, especially designed to perform $\mu \mathrm{SR}$ experiments under pressure [43-45]. As a pressure transmitting medium Daphne oil was used. The pressure was measured by tracking the superconducting transition of a very small indium plate by ac susceptibility. The filling factor of the pressure cell was maximized. The fraction of the muons stopping in the sample was approximately $40 \%$. The $\mu \mathrm{SR}$ time spectra were analyzed using the free software package MUSRFIT [46].

\section{RESULTS AND DISCUSSION}

\section{A. Crystal structure and phase purity}

We have synthesized the $\mathrm{Ca}_{1-x} \mathrm{Sr}_{x} \mathrm{AlSi}$ solid solution for $x=0,0.05,0.1,0.15,0.2,0.25,0.3,0.4,0.5,0.6,0.7,0.8$, 0.9 , and 1. All resulting products were found to be highly crystalline. For the end member $x=0$ of the solid solution, $\mathrm{CaAlSi}$, we were able to obtain high-quality single crystals 

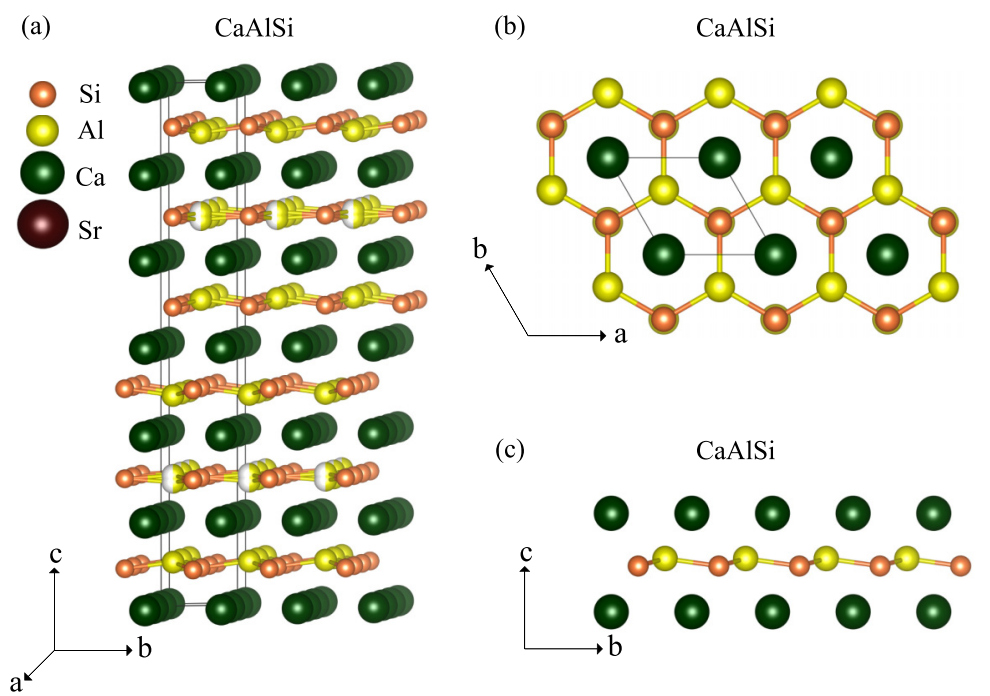

(d)

SrAlSi

(e)

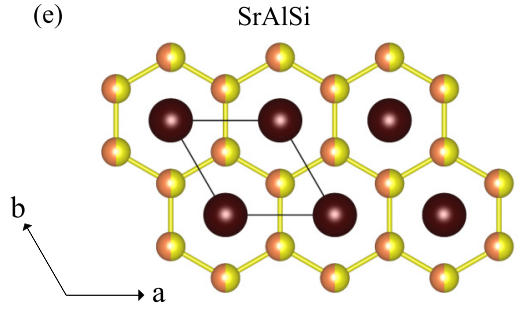

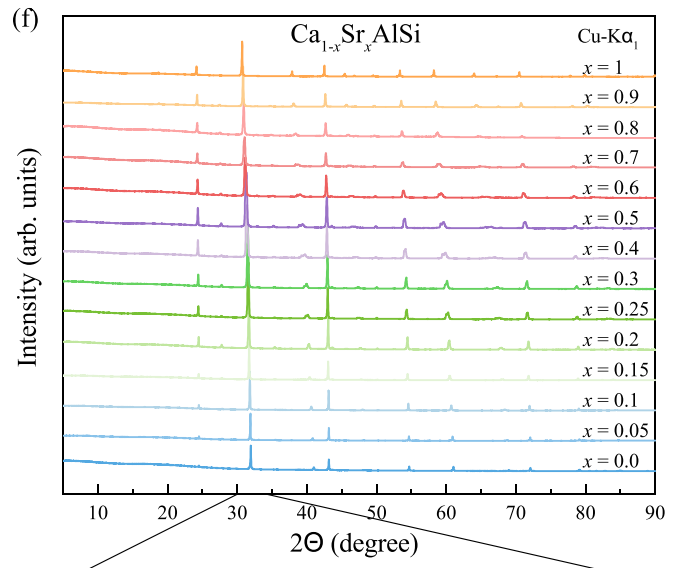

(g)

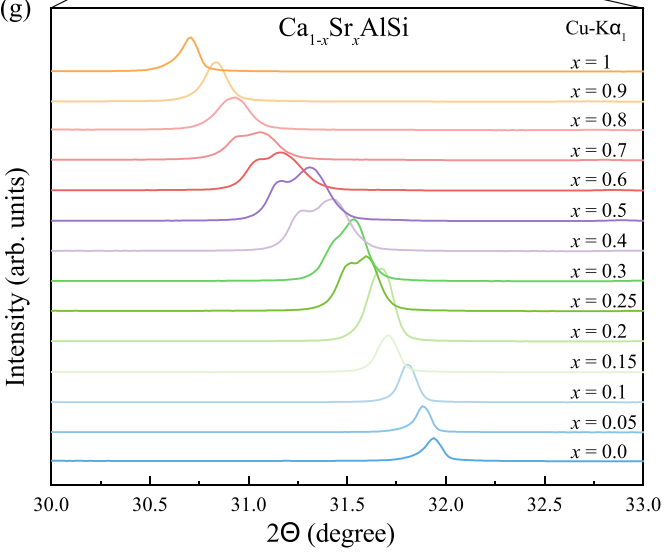

FIG. 1. Structure and bonding of the $\mathrm{Ca}_{1-x} \mathrm{Sr}_{x} \mathrm{AlSi}$ solid solution. Crystal structure of CaAlSi with space group $P 6_{3} / m m c$ (a) along the $a$ direction with an emphasis on the 6-layered super-cell, (b) along the $c$ direction, showing the honeycomb Al/Si layers, and (c) a single layer of CaAlSi showing the buckling of the Al/Si layers. Crystal structure of SrAlSi with space group P6/mmm in the AlB $\mathrm{B}_{2}$ structure type (d) along $a$ direction and (e) along $c$ direction. (f) Powder x-ray diffraction patterns of the $\mathrm{Ca}_{1-x} \mathrm{Sr}_{x} \mathrm{AlSi}$ solid solution with $x=0,0.05,0.1,0.15,0.2$, $0.25,0.3,0.4,0.5,0.6,0.7,0.8,0.9$, and $1(\mathrm{~g})$ Zoom-in of $2 \Theta=30.0$ to 33.0 region showing the pronounced shifting as a function of $\mathrm{Ca} / \mathrm{Sr}$ content and the slight splitting for the samples $x=0.3$ to 0.7 of the (101) Bragg reflection.

of up to sizes of $0.02 \times 0.04 \times 0.06 \mathrm{~mm}^{3}$ by this method. This allowed us to perform a detailed analysis of its crystal structure by means of SXRD. Earlier result from powder synchrotron and neutron diffraction experiments suggested that the unit cell of CaAlSi should to be 6-fold compared to the unit cell of the $\mathrm{AlB}_{2}$ structure [33]. Sagayama et al. had earlier developed a structural model, where they proposed that some of the honeycomb $\mathrm{Al} / \mathrm{Si}$ layers in CaAlSi are buckled, while others remained planar.

Our detailed analysis of the crystal structure shows now that: (i) The aluminum and silicon atoms are indeed not occupying random positions as it would be required for the $\mathrm{AlB}_{2}$ structure, and (ii) the unit cell is indeed 6-fold along the $c$ direction. (iii) Our data, however, unequivocally shows that all of the honeycomb $\mathrm{Al} / \mathrm{Si}$ layers are in fact buckled and that none of them remain planar, which is in contrary to earlier structural models. The aluminum atoms are thereby randomly distorted above and below the plain in the second and fifth layer of the unit cell. The distortion of the structure, which results in the buckling and different stacking of the $\mathrm{Al} / \mathrm{Si}$ layers result in the space group $P 6_{3} / \mathrm{mmc}$. It is worth noticing that the displacement within the $\mathrm{Al} / \mathrm{Si}$ layers in CaAlSi is small. The silicon atoms remaining in the plane and the aluminium atoms are displaced by $\Delta r$ of $0.173(11) \AA$. This structure resembles the well-known YPtAs-type structure with 4 hexagonal buckled layers, but the displacement of some of the aluminum atoms below the plane are causing CaAlSi to crystallize in its own, unique structure-type. The structure of CaAlSi with the unit-cell parameters of a $=4.1812(2) \AA$ and $c=26.3009(12) \AA$ is shown in Fig. 1 . In Fig. 1(b) the view along the $c$ direction is presented, which reveals how the hexagonal honeycomb Al/Si layers are stacked between layers of calcium atoms. The summary of the SXRD refinement are shown in the Table I and the details of the crystallographic positions are presented in Table II. The aluminium atoms occupy the $4 f$ Wyckoff position with occupancy 1 and 0.5 , respectively, for those atoms who display disorder. The calcium atoms occupy $2 a$ and $4 e$ positions, while Si atoms are located on the $4 f$ and $2 c$ positions.

We find that all other synthesized members of the $\mathrm{Ca}_{1-x} \mathrm{Sr}_{x} \mathrm{AlSi}$ solid solution crystallize in the $\mathrm{AlB}_{2}$-type structure with the $P 6 / \mathrm{mmm}$ space group, with random site occupancy in the $\mathrm{Al} / \mathrm{Si}$ and $\mathrm{Ca} / \mathrm{Sr}$ layers, respectively. These structures are confirmed by high-quality single-crystal data for samples with $x=0.2,0.4,0.5$, and 1 (the details of the refinements are listed in the Supplemental Material [47]). 
TABLE I. Details of the SXRD measurements and structural refinement for $\mathrm{CaAlSi}$

\begin{tabular}{|c|c|}
\hline \multicolumn{2}{|c|}{ Single crystal data for CaAlSi } \\
\hline Composition & CaAlSi \\
\hline CCDC/FIZ & CSD-2013753 \\
\hline Formula weight $[\mathrm{g} / \mathrm{mol}]$ & 285.45 \\
\hline Temperature $[\mathrm{K}]$ & $160(1)$ \\
\hline Crystal system & hexagonal \\
\hline Space group & $P 6_{3} / m m c$ \\
\hline$a[\AA]$ & $4.1812(2)$ \\
\hline$b[\AA]$ & $4.1812(2)$ \\
\hline$c[\AA]$ & $26.3009(12)$ \\
\hline$\alpha\left[{ }^{\circ}\right]$ & 90 \\
\hline$\beta\left[{ }^{\circ}\right]$ & 90 \\
\hline$\gamma\left[{ }^{\circ}\right]$ & 120 \\
\hline Volume $\left[\AA^{3}\right]$ & $398.20(4)$ \\
\hline $\mathrm{Z}$ & 2 \\
\hline$\rho_{\text {calc }}\left[\mathrm{g} / \mathrm{cm}^{3}\right]$ & 2.381 \\
\hline$\mu\left[\mathrm{mm}^{-1}\right]$ & 24.847 \\
\hline $\mathrm{F}(000)$ & 282.0 \\
\hline Crystal size $\left[\mathrm{mm}^{3}\right]$ & $0.02 \times 0.04 \times 0.06$ \\
\hline Radiation & $\mathrm{Cu}-\mathrm{K} \alpha(\lambda=1.54184 \AA)$ \\
\hline $2 \Theta$ range for data collection $\left[{ }^{\circ}\right]$ & 6.72 to 136.40 \\
\hline Index ranges & $\begin{array}{c}-5 \leqslant \mathrm{~h} \leqslant 4 \\
-4 \leqslant k \leqslant 5 \\
-31 \leqslant 1 \leqslant 31\end{array}$ \\
\hline Reflections collected & 181 \\
\hline Independent reflections & $\begin{array}{c}181\left[\mathrm{R}_{\mathrm{int}}=0.0082\right. \\
\left.\mathrm{R}_{\text {sigma }}=0.0036\right]\end{array}$ \\
\hline Data/restraints/parameters & $181 / 0 / 18$ \\
\hline Goodness-of-fit on $\mathrm{F}^{2}$ & 1.221 \\
\hline Final $R$ indexes $[I \geqslant 2 \sigma(I)]$ & $\begin{array}{c}\mathrm{R}_{1}=0.0153 \\
\mathrm{wR}_{2}=0.0517\end{array}$ \\
\hline Final $\mathrm{R}$ indexes [all data] & $\begin{array}{c}\mathrm{R}_{1}=0.0187 \\
\mathrm{w} \mathrm{R}_{2}=0.0545\end{array}$ \\
\hline Largest diff. peak/hole $\left[\mathrm{e} \AA^{-3}\right]$ & $0.23 /-0.16$ \\
\hline
\end{tabular}

The crystal structure, as a representative of the whole solid solution, of SrAlSi is depicted in Figs. 1(d) and 1(e) along the $a$ and the $c$ axis, respectively.

In Fig. 1(f) the PXRD pattern of all prepared members of the $\mathrm{Ca}_{1-x} \mathrm{Sr}_{x} \mathrm{AlSi}$ solid solution are shown. The analysis of the patterns reveals that all reflections shift systematically toward smaller $2 \Theta$ angles. This follows the expectation, as it corresponds to an increase of the unit cell parameters with

TABLE II. Atomic coordinates, isotropic displacement parameters, and occupancies of the atoms in CaAlSi obtained by SXRD.

\begin{tabular}{lcccclc}
\hline \hline \multicolumn{7}{c}{ Wyckoff positions } \\
\hline Atom & Symbol & $\mathrm{x}$ & $\mathrm{y}$ & $\mathrm{z}$ & \multicolumn{1}{c}{$\mathrm{U}_{\text {ISO }}$} & Occ. \\
\hline $\mathrm{A} 11$ & $4 f$ & $2 / 3$ & $1 / 3$ & $0.40928(4)$ & $0.0156(3)$ & 1 \\
$\mathrm{~A} 12$ & $4 f$ & $2 / 3$ & $1 / 3$ & $0.2566(4)$ & $0.0251(17)$ & 0.5 \\
$\mathrm{Ca} 1$ & $2 a$ & 0 & 0 & $1 / 2$ & $0.0132(3)$ & 1 \\
$\mathrm{Ca} 2$ & $4 e$ & 0 & 0 & $0.33227(2)$ & 0.0151 & 1 \\
$\mathrm{Si} 1$ & $4 f$ & $1 / 3$ & $2 / 3$ & $0.42205(3)$ & $0.0149(3)$ & 1 \\
$\mathrm{Si} 2$ & $2 c$ & $1 / 3$ & $2 / 3$ & $1 / 4$ & $0.0113(3)$ & 1 \\
\hline \hline
\end{tabular}

increasing strontium content. All calcium containing samples have small impurities of the calcium deficient phase $\mathrm{CaAl}_{2} \mathrm{Si}_{2}$ and the calcium-rich phase $\mathrm{Ca}_{3} \mathrm{Al}_{2} \mathrm{Si}_{2}$, accordingly. The impurities increase for synthesis attempts with alkaline-earth excesses, or upon annealing of the samples at various temperatures in quartz or niobium tubes.

A zoom-in of the PXRD patterns is show in Fig. 1(g) in the $30-33^{\circ} 2 \Theta$ range for the (101) Bragg reflection. The pronounced shifting of the (101) reflection can be clearly observed, furthermore a slight splitting of the reflection for $x$ values between 0.25 to 0.7 becomes apparent. The slight splitting is observed for the reflections (101), (201), (112), and (211). This is likely due to a lack of complete randomness in the distribution of calcium and strontium atoms along certain directions. It is unlikely that it is due to the presence of two phases with similar stoichiometries, because in this case two superconducting transitions close to each other would be expected. However, the superconducting transitions of this compositions are remarkably sharp (see discussion below). Reheating of the samples in the arc furnace removed the splitting but at the same time significantly increases the intensity of impurity peaks. Annealing and or reheating also substantially lowers the critical superconducting temperatures (see, Supplemental Material [47]). We have performed LeBail fits for all the compounds in order to obtain the respective cell parameters. The cell parameters $a$ and $c$ are found to change nearly linearly, following Vegard's law.

\section{B. Magnetic measurement}

The zero-field-cooled (ZFC) magnetization of all prepared members of the solid-solution $\mathrm{Ca}_{1-x} \mathrm{Sr}_{x} \mathrm{AlSi}$ with $x=0,0.05$, $0.1,0.15,0.2,0.25,0.3,0.4,0.5,0.6,0.7,0.8,0.9$, and 1 in an external field of $\mu_{0} H=2 \mathrm{mT}$ in a temperature range between $T=2 \mathrm{~K}$ and $10 \mathrm{~K}$ are depicted in Fig. 2. All magnetic shielding fractions were found to be larger than $100 \%$ for all the samples, due to demagnetization effects, confirming the bulk nature of the superconductivity in all samples. For better comparison, the magnetization data was normalized by plotting it as $-M(T) / M(2 \mathrm{~K})$. The transitions to the superconducting state are remarkably sharp for all the samples. The highest superconducting transition is observed for the parent compound of the solid solution $x=0$ (CaAlSi), with a critical temperature of $T_{\mathrm{c}} \approx 7.8 \mathrm{~K}$. This value corresponds to the highest critical temperature reported for any polymorph of CaAlSi earlier (see, e.g., Refs. [30,32,33]). We find the critical temperature to monotonically decrease with increasing strontium content, reaching a value of $T_{\mathrm{c}} \approx 4.9 \mathrm{~K}$ for SrAlSi $(x=1)$. The measured transition temperature for the prepared samples by arc melting of SrAlSi agrees well with previous reports (see, e.g., Refs. [25,30]). In Fig. 2, we have summarized the structural and superconducting parameters of the solid solution. It should be noted that the critical temperature in the solid solution does not follow the nearly linear change of the cell parameters, but that it has a more complex monotonic change as a function of strontium content. The critical temperature decreases more drastically close to the structurally distorted end member CaAlSi. This indicates that the higher $T_{\mathrm{c}}$ of $\mathrm{CaAlSi}$ and compositions close to it, are affected by an enhanced electron-phonon coupling due to a 

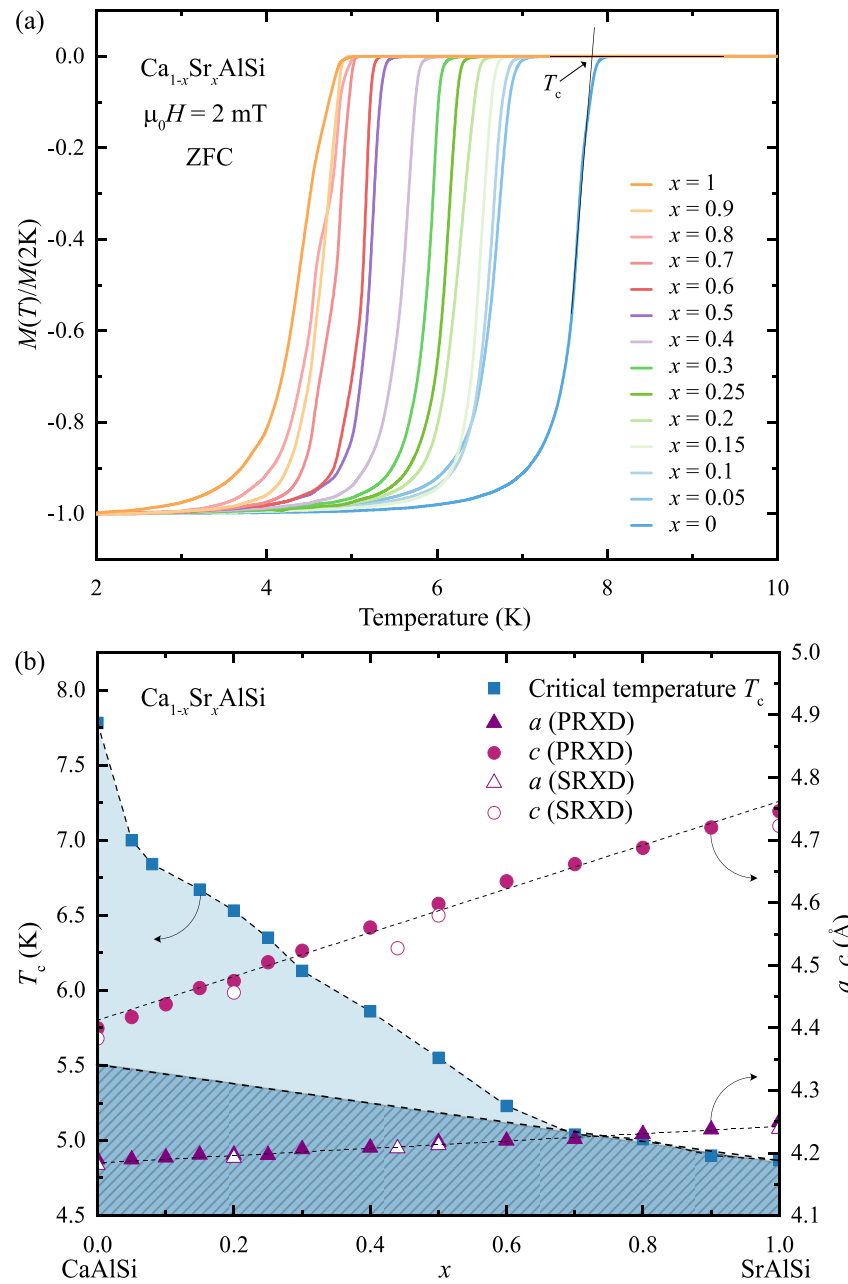

FIG. 2. Superconducting properties of the $\mathrm{Ca}_{1-x} \mathrm{Sr}_{x} \mathrm{AlSi}$ solidsolution. (a) ZFC magnetization in an external magnetic field of $\mu_{0} H=2 \mathrm{mT}$ for $x=0,0.05,0.1,0.15,0.2,0.25,0.3,0.4,0.5$, $0.6,0.7,0.8,0.9$, and 1 , plotted between 2 and $10 \mathrm{~K}$. Data were normalized by being plotted as $-M(T) / M(2 \mathrm{~K})$. (b) Summary of the crystallographic and superconducting parameters of the samples of the solid solution for $x=0,0.05,0.1,0.15,0.2,0.25,0.3,0.4,0.5$, $0.6,0.7,0.8,0.9$, and 1 . The cell parameter $c$ for CaAlSi was divided by 6 for comparability.

phonon-softening close to the structural instability. A mechanism that can emerge in superconductors close to structural phase transitions [1-4].

\section{Specific heat capacity}

We performed specific heat capacity measurements for the samples with $x=0,0.1,0.3,0.5$, and 1 in a temperature range of between $2 \mathrm{~K}$ to $16 \mathrm{~K}$. The specific heat capacity in the normal state was fitted using the following relationship:

$$
C / T=\gamma+\beta T^{2},
$$

where the Sommerfeld coefficient $\gamma$ corresponds to the electronic contribution, and $\beta$ corresponds to the phononic contribution to the total specific heat capacity. The Debye
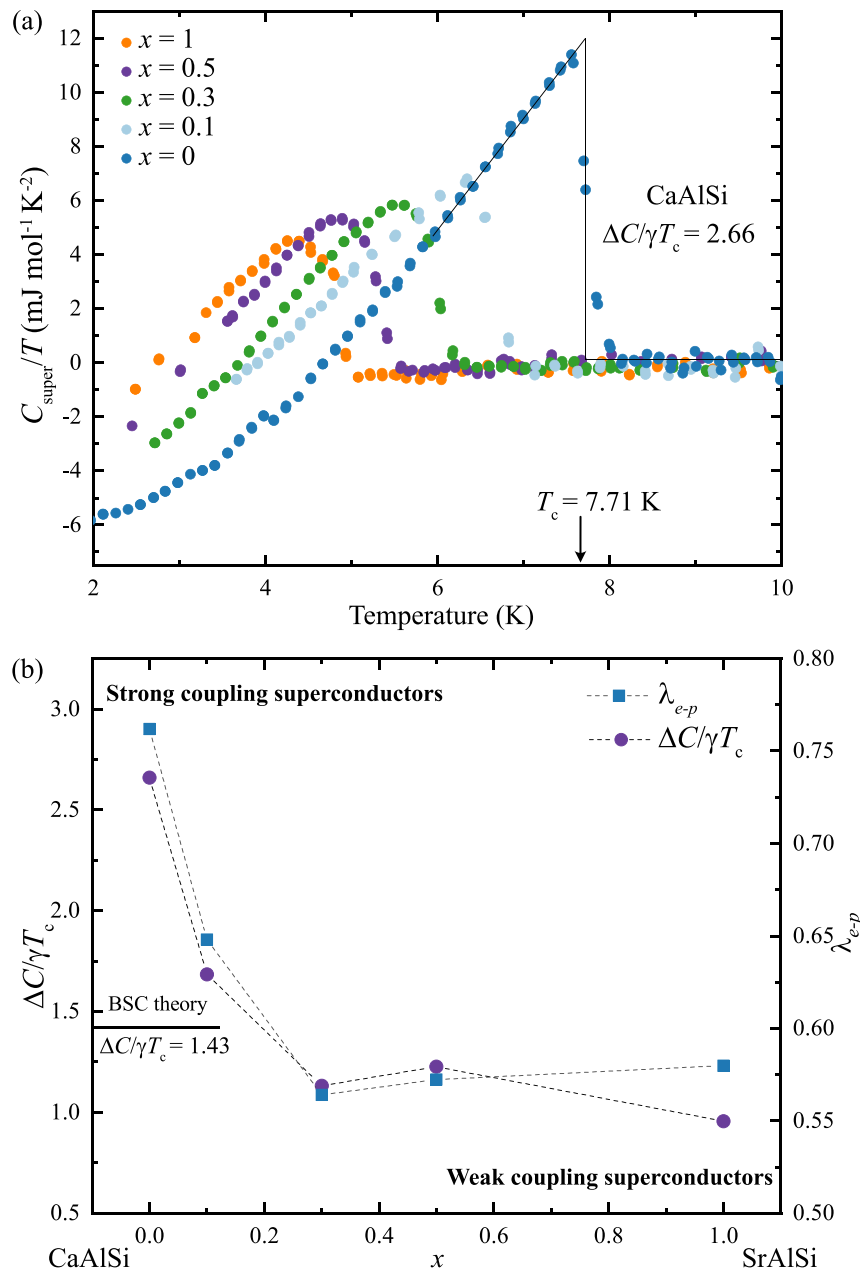

FIG. 3. (a) Specific heat capacity jump for the samples with $x=0,0.1,0.3,0.5$, and 1 in a temperature range of between $2 \mathrm{~K}$ to $10 \mathrm{~K}$. The entropy conserving construction is depicted for CaAlSi $(x=0)$. (b) Values of $\Delta C / \gamma T_{\mathrm{c}}$ and the electron-phonon coupling constant $\lambda_{e-p}$.

temperatures were calculated according to

$$
\Theta_{\mathrm{D}}=\left(\frac{12 \pi^{4}}{5 \beta} n R\right)^{1 / 3} .
$$

Here, $n=4$ is the number of atoms per formula unit and $\mathrm{R}$ $=8.31 \mathrm{~J} \mathrm{~mol}^{-1} \mathrm{~K}^{-1}$ is the gas constant. The obtained values for samples with $x=0,0.1,0.3,0.5$, and 1 are summarized in Table III. The obtained values for the end members CaAlSi and SrAlSi are in good agreement with earlier reports [25]. The values for $\gamma, \beta$, and $\Theta_{\mathrm{D}}$ change continuously across the solid solution.

The $C_{\text {super }}$ is shown in the Fig. 3(a), where the normal state specific heat capacity was subtracted $\left(C_{\text {super }} / T=C / T-\right.$ $\gamma-\beta T^{2}$ ) for better comparability of the results. The clearly observed discontinuities in the specific heat capacity at the superconducting transition temperatures confirm the bulk nature of the superconductivity in all samples. The entropy conserving construction that was used to determine the critical temperature $T_{\mathrm{c}}$ in the specific heat capacity and $\Delta C / \gamma T_{\mathrm{c}}$ is depicted for CaAlSi $(x=0)$. All values are summarized in 
TABLE III. Parameters obtained from specific heat capacity data for $\mathrm{Ca}_{1-x} \mathrm{Sr}_{x} \mathrm{AlSi}$ solid solution.

\begin{tabular}{|c|c|c|c|c|c|c|c|}
\hline \multicolumn{8}{|c|}{ Specific heat capacity data for $\mathrm{Ca}_{1-x} \mathrm{Sr}_{x} \mathrm{AlSi}$} \\
\hline & Parameter & $\gamma$ & $\beta$ & $\Theta_{\mathrm{D}}$ & $T_{\mathrm{c}}$ & $\Delta C / \gamma T_{\mathrm{c}}$ & $\lambda_{e-p}$ \\
\hline & & $\mathrm{mJ} \mathrm{mol}^{-1} \mathrm{~K}^{-2}$ & $\mathrm{~mJ} \mathrm{~mol}^{-1} \mathrm{~K}^{-4}$ & $\mathrm{~K}$ & $\mathrm{~K}$ & & \\
\hline $\mathrm{CaAlSi}$ & & $4.6(1)$ & $0.272(7)$ & 278 & 7.72 & 2.66 & 0.76 \\
\hline $\mathrm{Ca}_{0.9} \mathrm{Sr}_{0.1} \mathrm{AlSi}$ & & $4.78(9)$ & $0.104(5)$ & 383 & 6.66 & 1.74 & 0.65 \\
\hline $\mathrm{Ca}_{0.7} \mathrm{Sr}_{0.3} \mathrm{AlSi}$ & & $6.5(1)$ & $0.032(7)$ & 564 & 5.92 & 1.15 & 0.56 \\
\hline $\mathrm{Ca}_{0.5} \mathrm{Sr}_{0.5} \mathrm{AlSi}$ & & $5.75(8)$ & $0.056(5)$ & 471 & 5.24 & 1.27 & 0.57 \\
\hline SrAlSi & & $6.07(4)$ & $0.089(2)$ & 402 & 4.70 & 1.04 & 0.58 \\
\hline
\end{tabular}

Table III and in Fig. 3(b). As expected, the transition temperatures obtained from magnetization and specific heat capacity measurements, change in the same fashion, monotonically decreasing from $7.72 \mathrm{~K}$ to $4.70 \mathrm{~K}$. For CaAlSi $\Delta C / \gamma T_{\mathrm{c}}$ is found to be 2.66. This value is far beyond the value expected from BCS theory where it is predicted to be 1.43. $\Delta C / \gamma T_{\mathrm{c}}$ decreases across the solid solution to a value of 1.04 for $\operatorname{SrAlSi}(x=1)$. The electron-phonon coupling constant $\lambda_{e-p}$ can be estimated from the specific heat capacity using the McMillan formula [48]:

$$
\lambda_{e-p}=\frac{1.04+\mu^{*} \ln \left(\Theta_{\mathrm{D}} / 1.45 T_{\mathrm{c}}\right)}{\left(1-0.62 \mu^{*}\right) \ln \left(\Theta_{\mathrm{D}} / 1.45 T_{\mathrm{c}}\right)-1.04} .
$$

Here, we used $\mu^{*}=0.13$ for the Coulomb pseudopotential parameter, as commonly done for intermetallics [49]. We find $\lambda_{e-p}$ to decrease from 0.76 for CaAlSi, to 0.58 for SrAlSi by this analysis.

Our study of the specific heat capacity of the $\mathrm{Ca}_{1-x} \mathrm{Sr}_{x} \mathrm{AlSi}$ solid solution confirms the bulk nature of the superconductivity in all samples and that superconductivity in CaAlSi is best described in the strong coupling limit while SrAlSi is best described in the weak coupling limit.

\section{London magnetic penetration depth}

We have used $\mu \mathrm{SR}$ measurements in order to investigate the London magnetic penetration depth in a temperature range between $T=300 \mathrm{mK}$ and $10 \mathrm{~K}$. In $\mu$ SR experiments nearly $100 \%$ spin-polarized muons $\mu^{+}$are implanted into the investigated sample one at a time. The positively charged $\mu^{+}$ thermalize at interstitial crystallographic sites, where they act as magnetic microprobes. In a magnetic material the muon spin precesses in the local field $B_{\mu}$ at the muon site with the Larmor frequency:

$$
v_{\mu}=\gamma_{\mu} /(2 \pi) B_{\mu} .
$$

For the muon the gyromagentic ratio is $\gamma_{\mu} /(2 \pi)=$ $135.5 \mathrm{MHz} \mathrm{T}^{-1}$. Using the $\mu \mathrm{SR}$ technique fundamental microscopic parameters of superconductors can be measured, namely the magnetic penetration depth $\lambda$ and the coherence length $\xi[50]$.

If a type-II superconductor is cooled below critical temperature $T_{\mathrm{c}}$ in an applied magnetic field a vortex lattice is formed, which in general is incommensurate with the crystal lattice with vortex cores separated by much larger distances than those of the unit cell. Because the implanted muons stop at given crystallographic sites, they will randomly probe the field distribution of the vortex lattice. These measurements are performed in a field applied perpendicular to the initial muon spin polarization (so called TF configuration) [50-52].

In Fig. 4, we present transverse-field (TF- $\mu \mathrm{SR})$ time spectra measured above $(10 \mathrm{~K})$ and below $(1.5 \mathrm{~K})$ the superconducting transition temperature of (a) CaAlSi $(x=0)$, (b) $\mathrm{Ca}_{0.7} \mathrm{Sr}_{0.3} \mathrm{AlSi}(x=0.3)$, and (c) SrAlSi $(x=1)$, respectively. For all measured samples, oscillations at $10 \mathrm{~K}$ occur at a very small relaxation with time. This very small relaxation is due to random local magnetic fields within the samples. For the time spectra at $1.5 \mathrm{~K}$ the oscillations show a significant decrease due to the formation of the flux-line lattice (FLL), and thus nonuniform magnetic field distribution in the Shubnikov phase. The solid lines represent fits to the data points by using the following functional form [46]

$$
P_{s}(t)=\sum_{i=1}^{2} A_{s, i} \exp \left[-\frac{\sigma_{i}^{2} t^{2}}{2}\right] \cos \left(\gamma_{\mu} B_{\text {int }, s, i} t+\varphi\right) .
$$

Two component expression was used for the sample due to the observed weak asymmetric field distribution. In Eq. (5), $A_{\mathrm{s}, \mathrm{i}}, B_{\text {int,i }}$, and $\sigma_{\mathrm{i}}$ are the asymmetry, the mean field and the relaxation rate of the $i$ th component, and $\varphi$ is the initial phase of the muon-spin ensemble. $\gamma /(2 \pi) \simeq 135.5 \mathrm{MHz} \mathrm{\textrm {T } ^ { - 1 }}$ is the muon gyromagnetic ratio. In order to extract the second moment of the field distribution from the two component fitting we used a similar procedure as described in Ref. [53]. To analyze the data, obtained under hydrostatic pressure, the following function was added:

$$
P_{p c}(t)=A_{p c} \exp \left[-\frac{\sigma_{p c}^{2} t^{2}}{2}\right] \cos \left(\gamma_{\mu} B_{\text {int }, p c} t+\varphi\right),
$$

Here $A_{\mathrm{pc}}$ denote the initial asymmetry of the pressure cell. The Gaussian relaxation rate, $\sigma_{\mathrm{pc}}$, reflects the depolarization due to the nuclear moments of the pressure cell. The width of the pressure cell signal increases below the critical temperature $T_{\text {c }}$. As shown previously [44], this is due to the influence of the diamagnetic moment of the superconducting sample on the pressure cell, leading to the temperature dependent $\sigma_{\mathrm{pc}}$ below the critical temperature $T_{\mathrm{c}}$. In order to consider this influence, we assume the linear coupling between $\sigma_{\mathrm{pc}}$ and the field shift of the internal magnetic field in the superconducting state: $\sigma_{\mathrm{pc}}(T)=\sigma_{\mathrm{pc}}\left(T>T_{\mathrm{c}}\right)+C(T)\left(\mu_{0} H_{\mathrm{int}, \mathrm{NS}}-\mu_{0} H_{\mathrm{int}, \mathrm{SC}}\right)$, where $\sigma_{\mathrm{pc}}\left(T>T_{\mathrm{c}}\right)=0.25 \mu \mathrm{s}^{-1}$ is the temperature independent Gaussian relaxation rate. $\mu_{0} H_{\text {int,NS }}$ and $\mu_{0} H_{\text {int,SC }}$ are the internal magnetic fields measured in the normal and in the 

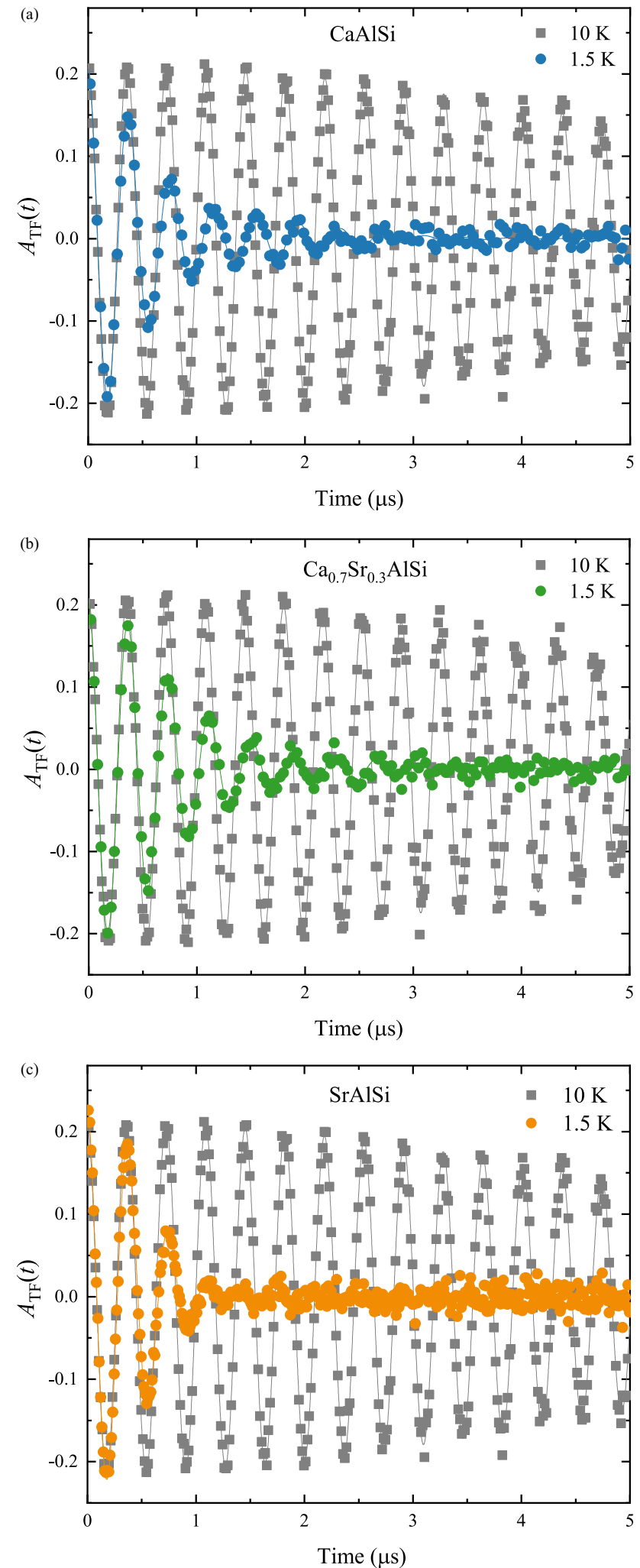

FIG. 4. Transverse-field $\mu \mathrm{SR}$ time spectra of the $\mathrm{Ca}_{1-x} \mathrm{Sr}_{x} \mathrm{AlSi}$ solid solution. (a) CaAlSi, (b) $\mathrm{Ca}_{0.7} \mathrm{Sr}_{0.3} \mathrm{AlSi}$, and (c) SrAlSi spectra in the normal state at $T=10 \mathrm{~K}$ (gray points) and in the superconducting state at $T=1.5 \mathrm{~K}$ (blue, green, and orange points, respectively). The solid lines represent fits to the data points by means of Eq. (5).

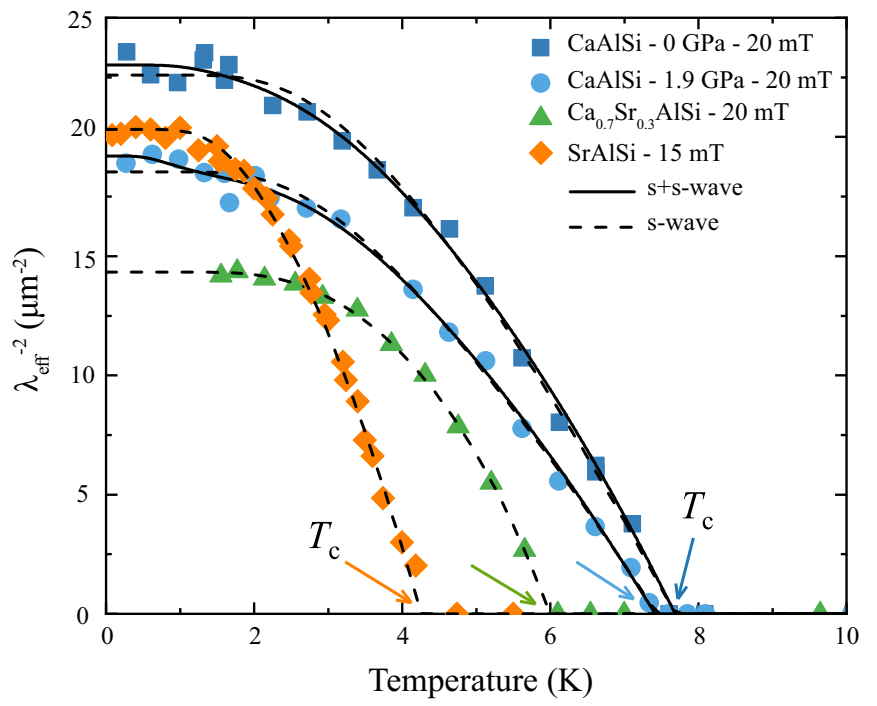

FIG. 5. Temperature evolution of the London magnetic penetration depth $\lambda^{-2}(T)$ for $\mathrm{Ca}_{1-x} \mathrm{Sr}_{x} \mathrm{AlSi}$. The temperature dependence of the inverse squared magnetic penetration depth $\lambda^{-2}$ for CaAlSi (blue), $\mathrm{Ca}_{0.7} \mathrm{Sr}_{0.3} \mathrm{AlSi}$ (green), and $\mathrm{SrAlSi}$ (orange), measured at ambient and under pressure in a temperature range between $T=$ $300 \mathrm{mK}$ and $10 \mathrm{~K}$. Dashed line represents a fit with the single gap $s$-wave model, while the solid line corresponds to the two-gap $s+s$ wave model.

superconducting state, respectively. The TF- $\mu$ SR spectra of all samples show a strong relaxation of the signal below $T_{\mathrm{c}}$, which is evidence for the bulk type-II superconductivity in these compounds and also for CaAlSi under $p=1.9 \mathrm{GPa}$.

From the TF- $\mu$ SR spectra of $\mathrm{Ca}_{1-x} \mathrm{Sr}_{x}$ AlSi with $x=0$, 0.3 , and 1 , the superconducting part of the square root of the second moment of the field distribution in the vortex state $[51,54]\left(\sqrt{\left\langle\Delta B^{2}\right\rangle} \propto \sigma_{\mathrm{sc}} \propto \lambda_{\text {eff }}^{-2}\right)$ was obtained by subtracting the nuclear moment contribution $\left(\sigma_{\mathrm{nm}}\right)$ measured at $T>T_{\mathrm{c}}$ according to $\sigma_{\mathrm{sc}}^{2}=\sigma^{2}-\sigma_{\mathrm{nm}}^{2} . \sigma_{\mathrm{sc}}$ is the Gaussian relaxation rate due to the formation of the FLL [51]. In the analysis $\sigma_{\mathrm{nm}}$ was assumed to be constant over the entire temperature range.

In order to investigate the symmetry of the superconducting gap, we have therefore derived the temperature-dependent London magnetic penetration depth $\lambda(T)$, which is related to the relaxation rate by

$$
\frac{\sigma_{\mathrm{sc}}(T)}{\gamma_{\mu}}=0.06091 \frac{\Phi_{0}}{\lambda^{2}(T)} .
$$

Here, $\gamma_{\mu}$ is the gyromagnetic ratio of the muon, and $\Phi_{0}$ is the magnetic-flux quantum. In accordance with the convention in the field, the magnetic penetration depth is provided as $\lambda^{-2}$ throughout the paper in order to emphasis the relationship $\rho_{S} \propto \lambda^{-2}$, with $\rho_{S}$ being the superfluid density.

In Fig. 5, we show the temperature dependence of the $\lambda^{-2}$ for $\mathrm{Ca}_{1-x} \mathrm{Sr}_{x} \mathrm{AlSi}$ with $x=0,0.3$, and 1 at ambient pressure. In addition, the $\lambda^{-2}(T)$ is shown for CaAlSi under the maximum applied pressure of $p=1.9 \mathrm{GPa}$, using a high-pressure piston-cylinder set-up [43]. $\lambda(T)$ was calculated within the local (London) approximation $(\lambda \gg \xi)$ by the following 

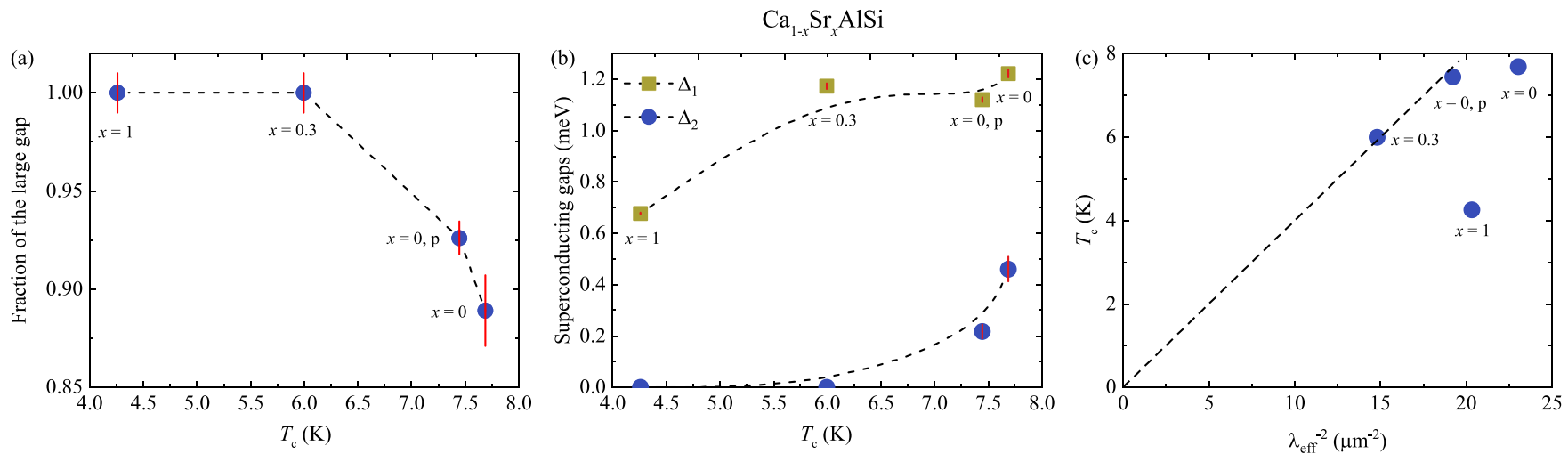

FIG. 6. Superconducting gap evolution as a function of the critical temperature. Details of the analysis of $\lambda^{-2}$ and $\Delta_{0}$ for $x=0,0.3$, and 1 with (a) the fraction of the large gap vs $T_{\mathrm{c}}$, (b) the size of the superconducting gaps vs $T_{\mathrm{c}}$, and (c) the critical temperature $T_{\mathrm{c}}$ against the $\lambda_{\text {eff }}^{-2}(0)$ obtained from the $\mu$ SR experiments. In (a) and (b) the dotted line is a guide for the eye, while in (c) it represents the expected behavior according to the BCS weak-coupling limit.

expression $[46,55]$ :

$\frac{\lambda^{-2}\left(T, \Delta_{0, i}\right)}{\lambda^{-2}\left(0, \Delta_{0, i}\right)}=1+\frac{1}{\pi} \int_{0}^{2 \pi} \int_{\Delta(T, \varphi)}^{\infty}\left(\frac{\partial f}{\partial E}\right) \frac{E d E d \varphi}{\sqrt{E^{2}-\Delta_{i}(T, \varphi)^{2}}}$,

where $f=\left[1+\exp \left(E / k_{B} T\right)\right]^{-1}$ is the Fermi function, $\varphi$ is the angle along the Fermi surface, and $\Delta_{i}(T, \varphi)=$ $\Delta_{0, i} \Gamma\left(T / T_{\mathrm{c}}\right) g(\varphi)\left(\Delta_{0, i}\right.$ is the maximum gap value at $\left.T=0\right)$. The temperature dependence of the gap is approximated by the expression $\Gamma\left(T / T_{\mathrm{c}}\right)=\tanh \left\{1.82\left[1.018\left(T_{\mathrm{c}} / T-1\right)\right]^{0.51}\right\}$ [56], while $g(\varphi)$ describes the angular dependence of the gap and it is replaced by 1 for both an $s$-wave and an $s+s$-wave gap. The results of the gap analysis are shown in Fig. 5 .

The form of the temperature dependence of $\lambda^{-2}$ shows saturation at low temperatures for all 3 samples and also for CaAlSi at $1.9 \mathrm{GPa}$. Thus, the flat $T$-dependence of $\lambda^{-2}$ observed in $\mathrm{Ca}_{1-x} \mathrm{Sr}_{x} \mathrm{AlSi}$ for low temperatures is consistent with a nodeless superconductor, in which $\lambda^{-2}(T)$ reaches its zero-temperature value exponentially.

We find that the London magnetic penetration depth $\lambda^{-2}(T)$ for $\mathrm{CaAlSi}$, at ambient pressure, as well as at a pressure of $p=1.9 \mathrm{GPa}$, are best described by a two-gap $s+s$-wave symmetric superconducting gap (solid black line). Both the ambient pressure, as well as the $p=1.9 \mathrm{GPa}$ measurements are insufficiently described by a single-gap $s$-wave symmetric gap (dotted line). The two-gap $i+i$-wave scenario-with the large gap of CaAlSi being 1.174(7) meV and the smaller gap being $0.24(2) \mathrm{meV}$-describes the experimental data remarkably well. The conclusion for a two-gap scenario is supported by the commonly used $\chi^{2}$-test $[57,58]$. In our case, the value of $\chi^{2}$ for CaAlSi is for a single gap $i$-wave model $15 \%$ higher than the for the two-gap $i$ wave model. The two-gap scenario is furthermore in good agreement with earlier field-dependant $\mu \mathrm{SR}$ measurements [35].

The London magnetic penetration depth $\lambda^{-2}(T)$ for the samples $\mathrm{Ca}_{0.7} \mathrm{Sr}_{0.3} \mathrm{AlSi}$ and $\mathrm{SrAlSi}$ are, on the other hand, best described by the single-gap $s$-wave model. These results strongly indicate that strontium-doping not only stabilizes the simpler $\mathrm{AlB}_{2}$-structure, and reduces the critical temper- ature, but also shifts the system from two-gap to single-gap superconductivity. Our results show that the lower critical temperatures $T_{\mathrm{c}}$ are anticorrelated with two-gap $s+s$-wave superconductivity and that there is a critical value of $T_{\mathrm{c}}$, which separates two types of superconductivity in the solid solution of $\mathrm{Ca}_{1-x} \mathrm{Sr}_{x} \mathrm{AlSi}$. Noteworthy is also the effect of the pressure for the CaAlSi sample. Applying 1.9 GPa has a big effect on the superfluid density, $\lambda_{\text {eff }}^{-2}$, which changes from $23.0 \mu \mathrm{m}^{-2}$ to $19.1 \mu \mathrm{m}^{-2}$ under pressure, whereas it has a small effect on the transition temperature (change from $7.7 \mathrm{~K}$ to $7.4 \mathrm{~K}$ ).

In Figs. 6(a) and 6(b), we show the fraction of the large gap and the values of large superconducting gap $\Delta_{1}$ as well as small gap $\Delta_{2}$, respectively, as a function of $T_{\mathrm{c}}$. These results suggest the critical value for $T_{\mathrm{c}, \mathrm{cr}} \simeq 6 \mathrm{~K}$, above which the fraction of the second superconducting gap with the gap value of $\Delta_{2}=0.2 \mathrm{meV}$ starts to evolve. Hence, the substitution of calcium with strontium and the application of pressure can be interpreted as tuning parameters that shift the critical temperature $T_{\mathrm{c}}$ below the critical value, and move the system towards a single gap superconductivity. In Fig. 6(c) the critical temperature $T_{\mathrm{c}}$ against the $\lambda^{-2}(0)$ obtained from the $\mu \mathrm{SR}$ experiments is shown in a so-called Uemura plot. Within the picture of the Bose-Einstein condensate (BEC) to BCS crossover, systems exhibiting small $T_{\mathrm{c}} / T_{F}$ (with $T_{F}$ being the Fermi temperature) are considered to be in the BCS-like side, while the linear relationship between $T_{\mathrm{c}}$ and $T_{F}$ is expected only in the BEC-like side. The linear and nonmonotonous relationships have been used in the past for the characterization of BCS-like, so-called conventional superconductors and BEC-like, so-called unconventional superconductors. The present results on $\mathrm{Ca}_{1-x} \mathrm{Sr}_{x} \mathrm{AlSi}$ demonstrate that a nonmonotoneuous relation between $T_{\mathrm{c}}$ and the superfluid density $\rho_{S}=$ $n_{s} / m^{*}$ (where $n_{s}$ is the superconducting carrier density and $m^{*}$ is the effective mass) holds for this system [59,60]. This system falls into the clean limit, and therefore, such a behavior is unrelated to pair breaking. It may be worthwhile to mention that the values observed are far off to the right-hand side of the Uemura line, i.e., their condensate densities are similar to the ones of optimal doped cuprates whereas the critical temperatures are much lower here. In the unlikely case that the mixture, in contrary to the end members of the solid solution, 
are in the dirty limit: Then the reduction of the condensate density of the sample $x=0.3$ could be caused by the Ca vs $\mathrm{Sr}$ disorder [61,62].

Besides the transition between $s$ - to $s+s$-wave superconductivity, another important observation from this data is that there is an unconventional effect on the superfluid density $\rho_{S}$ by strontium doping and the application of pressure. This becomes apparent when we compare the extrapolated values of the London magnetic penetration depth at absolute zero $\lambda_{\text {eff }}^{-2}(0)$ with each other. $\lambda_{\text {eff }}^{-2}(0)$ decreases from a value of $23.0 \mu \mathrm{m}^{-2}$ for CaAlSi with $T_{\mathrm{c}}=7.7 \mathrm{~K}$ to $14.3 \mu \mathrm{m}^{-2}$ for $\mathrm{Ca}_{0.7} \mathrm{Sr}_{0.3} \mathrm{AlSi}$ with $T_{\mathrm{c}}=6.0 \mathrm{~K}$. The correlation between $\lambda_{\text {eff }}^{-2}$ and $T_{\mathrm{c}}$ is consistent with the behavior observed in other unconventional two-gap superconductors [63-67]. However, for $\mathrm{SrAlSi}$, in which $T_{\mathrm{c}}$ is further reduced to $4.2 \mathrm{~K}$, then $\lambda_{\text {eff }}^{-2}(0)$ significantly increases to $20.3 \mu \mathrm{m}^{-2}$. This means that for SrAlSi, a much higher superfluid density is observed than it is expected from the value of the critical temperature, which is normally the case for conventional superconductors (compare, e.g., Refs. [68,69]). This nonmonotonous behavior of $\lambda_{\text {eff }}^{-2}(0)$ and its strong enhancement for SrAlSi supports the idea that the nature of superconductivity changes depending whether the compound displays a two-gap or single-gap superconducting state.

\section{SUMMARY AND CONCLUSION}

We successfully synthesized the $\mathrm{Ca}_{1-x} \mathrm{Sr}_{x} \mathrm{AlSi}$ solid solution with $x=0,0.05,0.1,0.15,0.2,0.25,0.3,0.40 .5$, $0.6,0.7,0.8,0.9$, and 1 . We have analyzed the structure and phase purity by $\mathrm{x}$-ray diffraction. We show by employing single-crystal $\mathrm{x}$-ray diffraction that the structure of CaAlSi consists of a 6-folded unit cell along the $c$ axis with none of the honeycomb Al/Si layers being planar, but all of them slightly buckled. Hence, CaAlSi crystallizes in its own unique structure in the space group $P 6_{3} / m m c$. We have found all other members of the solid solution to crystallize in the $\mathrm{AlB}_{2}$ type structure with random site occupancies in the $\mathrm{Al} / \mathrm{Si}$ and $\mathrm{Ca} / \mathrm{Sr}$ layers, respectively. We have, furthermore, shown that all members of the solid solution are bulk superconductors with monotonically varying critical temperatures $T_{\mathrm{c}}$ by means of magnetization measurements. The highest superconducting transition is observed for the parent compound of the solid solution $x=0(\mathrm{CaAlSi})$, with a critical temperature of $T_{\mathrm{c}} \approx$ $7.8 \mathrm{~K}$. We find the critical temperature to monotonically decrease with increasing strontium content, reaching a value of $T_{\mathrm{c}} \approx 4.9 \mathrm{~K}$ for $x=1$ (SrAlSi). We found that while the cell parameters $a$ and $c$ change across the solid solution nearly linearly, following Vegard's law, the critical temperature $T_{\mathrm{c}}$ has a more complex change as a function of strontium content. The critical temperature decreases more drastically close to the structurally distorted end member CaAlSi. This indicates that the higher $T_{\mathrm{c}}$ of CaAlSi and compositions close to it, might be affected by an enhanced electron-phonon coupling due to a phonon-softening close to the structural instability. Our specific heat capacity measurements confirm the bulk nature of the superconductivity in all members of the solid solution. These measurements further indicate the different character of the superconductivity by a large change in the values for $\Delta C / \gamma T_{\mathrm{c}}$ from 2.66 to 1.04 across the solid solution.

This finding is further supported by an analysis of the microscopic superconducting properties of the solid solution namely the measurements of the London magnetic penetration depth by means of $\mu \mathrm{SR}$ measurements. We have shown that SrAlSi possesses a magnetic penetration depth $\lambda_{\text {eff }}^{-2}(0)$ of $20.3 \mu \mathrm{m}^{-2}$, while for $\mathrm{Ca}_{0.7} \mathrm{Sr}_{0.3} \mathrm{AlSi}$ it is equal to $14.3 \mu \mathrm{m}^{-2}$. Both compounds are one gap superconductors with gaps of $\Delta_{0}=0.68 \mathrm{meV}$ and $1.17 \mathrm{meV}$, respectively. CaAlSi has a magnetic penetration depth of $23.0 \mu \mathrm{m}^{-2}$ and is a two gap superconductor with gaps of $\Delta_{1}=1.22 \mathrm{meV}$ and $\Delta_{2}=0.46 \mathrm{meV}$. The London penetration depth changes to $19.1 \mu \mathrm{m}^{-2}$ and gaps to $\Delta_{1}=1.12 \mathrm{meV}$ and $\Delta_{2}=0.22 \mathrm{meV}$, respectively, under $1.9 \mathrm{GPa}$ pressure, which indicates that pressure pushes system towards the one gap model. We find that the subtle change in the crystal structure, i.e., the buckling of the layers in $\mathrm{CaAlSi}$, does not only enhance the critical temperature in the $\mathrm{Ca}_{1-x} \mathrm{Sr}_{x} \mathrm{AlSi}$ solid solution substantially, but that it also initiates a single-gap to two-gap superconducting transition, leading to unconventional superconducting properties in the end member of the solid solution. The London magnetic penetration depth $\lambda^{-2}(T)$ for CaAlSi, at $p=$ $0 \mathrm{GPa}$ and $1.9 \mathrm{GPa}$, is well described by a two-gap $s+s$ wave model, while $\lambda^{-2}(T)$ for the samples $\mathrm{Ca}_{0.7} \mathrm{Sr}_{0.3} \mathrm{AlSi}$ and SrAlSi are compatible with the single-gap $s$-wave model. We have shown that the $\mathrm{Ca}_{1-x} \mathrm{Sr}_{x} \mathrm{AlSi}$ is a rich superconducting system, where a structural transition and a two-gap to single-gap superconducting transition can be controlled by a isoelectronic chemical substitution, or by pressure, making this a promising model system for the investigation of the entanglement of structural and electronic interactions in superconductors on honeycomb lattices. Our results may contribute to a better understanding of structure-compositionproperty relations in layered superconducting materials in general.

\section{ACKNOWLEDGMENTS}

We thank Markus Bendele for experimental help during the beginning of this project, and Andreas Schilling for helpful discussions. Energy-dispersive x-ray spectroscopy was performed with equipment maintained by the Center for Microscopy and Image Analysis at the University of Zurich. This work was supported by the Swiss National Science Foundation under Grants No. PZ00P2_174015 and No. PCEFP2_194183.
[1] J. Slusky, N. Rogado, K. Regan, M. Hayward, P. Khalifah, T. He, K. Inumaru, S. Loureiro, M. Haas, H. Zandbergen et al., Loss of superconductivity with the addition of $\mathrm{Al}$ to $\mathrm{MgB}_{2}$ and a structural transition in $\mathrm{Mg}_{1-\mathrm{x}} \mathrm{Al}_{\mathrm{x}} \mathrm{B} 2$, Nature (London) 410 , 343 (2001).
[2] D. Hirai, F. von Rohr, and R. J. Cava, Emergence of superconductivity in $\mathrm{BaNi}_{2}\left(\mathrm{Ge}_{1-x} \mathrm{P}_{x}\right)_{2}$ at a structural instability, Phys. Rev. B 86, 100505(R) (2012).

[3] M. Lang, R. Kürsch, A. Grauel, C. Geibel, F. Steglich, H. Rietschel, T. Wolf, Y. Hidaka, K. Kumagai, Y. Maeno et al., 
Lattice Instabilities in Cuprate Superconductors: A Possible Limiting Mechanism for $T_{c}$, Phys. Rev. Lett. 69, 482 (1992).

[4] M. S. Torikachvili, S. L. Budko, N. Ni, and P. C. Canfield, Pressure Induced Superconductivity in $\mathrm{CaFe}_{2} \mathrm{As}_{2}$, Phys. Rev. Lett. 101, 057006 (2008).

[5] P. B. Allen and M. L. Cohen, Superconductivity and Phonon Softening, Phys. Rev. Lett. 29, 1593 (1972).

[6] X. Wu, H. O. Jeschke, D. Di Sante, F. O. von Rohr, R. J. Cava, and R. Thomale, Origin of the pressure-dependent $T_{c}$ valley in superconducting simple cubic phosphorus, Phys. Rev. Materials 2, 034802 (2018).

[7] L. R. Testardi, Structural instability and superconductivity in A-15 compounds, Rev. Mod. Phys. 47, 637 (1975).

[8] D. G. Hinks, D. R. Richards, B. Dabrowski, D. T. Marx, and A. W. Mitchell, The oxygen isotope effect in $\mathrm{Ba}_{0.625} \mathrm{~K}_{0.375} \mathrm{BiO}_{3}$, Nature (London) 335, 419 (1988).

[9] A. Gauzzi, S. Takashima, N. Takeshita, C. Terakura, H. Takagi, N. Emery, C. Hérold, P. Lagrange, and G. Loupias, Enhancement of Superconductivity and Evidence of Structural Instability in Intercalated Graphite $\mathrm{CaC}_{6}$ under High Pressure, Phys. Rev. Lett. 98, 067002 (2007).

[10] Y. Cao, V. Fatemi, S. Fang, K. Watanabe, T. Taniguchi, E. Kaxiras, and P. Jarillo-Herrero, Unconventional superconductivity in magic-angle graphene superlattices, Nature (London) 556, 43 (2018).

[11] J. Nagamatsu, N. Nakagawa, T. Muranaka, Y. Zenitani, and J. Akimitsu, Superconductivity at $39 \mathrm{~K}$ in magnesium diboride, Nature (London) 410, 63 (2001).

[12] J. Bardeen, L. N. Cooper, and J. R. Schrieffer, Theory of superconductivity, Phys. Rev. 108, 1175 (1957).

[13] I. Mazin and V. Antropov, Electronic structure, electron-phonon coupling, and multiband effects in $\mathrm{MgB}_{2}$, Physica C 385, 49 (2003).

[14] S. L. Budko, G. Lapertot, C. Petrovic, C. E. Cunningham, N. Anderson, and P. C. Canfield, Boron Isotope Effect in Superconducting $\mathrm{MgB}_{2}$, Phys. Rev. Lett. 86, 1877 (2001).

[15] T. E. Weller, M. Ellerb, S. S. Saxena, R. P. Smith, and N. T. Skipper, Superconductivity in the intercalated graphite compounds $\mathrm{C}_{6} \mathrm{Yb}$ and $\mathrm{C}_{6} \mathrm{Ca}$, Nat. Phys. 1, 39 (2005).

[16] I. I. Mazin, Intercalant-Driven Superconductivity in $\mathrm{C}_{6} \mathrm{Yb}$ and $\mathrm{C}_{6}$ Ca, Phys. Rev. Lett. 95, 227001 (2005).

[17] M. Calandra and F. Mauri, Theoretical Explanation of Superconductivity in $\mathrm{C}_{6} \mathrm{Ca}$, Phys. Rev. Lett. 95, 237002 (2005).

[18] G. Csányi, P. B. Littlewood, A. H. Nevidomskyy, C. J. Pickard, and B. D. Simons, The role of the interlayer state in the electronic structure of superconducting graphite intercalated compounds, Nat. Phys. 1, 42 (2005).

[19] Y. Nishikubo, K. Kudo, and M. Nohara, Superconductivity in the honeycomb-lattice pnictide SrPtAs, J. Phys. Soc. Jpn. 80, 055002 (2011).

[20] P. K. Biswas, H. Luetkens, T. Neupert, T. Stürzer, C. Baines, G. Pascua, A. P. Schnyder, M. H. Fischer, J. Goryo, M. R. Lees, H. Maeter, F. Brckner, H.-H. Klauss, M. Nicklas, P. J. Baker, A. D. Hillier, M. Sigrist, A. Amato, and D. Johrendt, Evidence for superconductivity with broken time-reversal symmetry in locally noncentrosymmetric SrPtAs, Phys. Rev. B 87, 180503(R) (2013).

[21] M. H. Fischer, T. Neupert, C. Platt, A. P. Schnyder, W. Hanke, J. Goryo, R. Thomale, and M. Sigrist, Chiral d-wave superconductivity in SrPtAs, Phys. Rev. B 89, 020509 (2014).
[22] M. Imai, K. Nishida, T. Kimura, and H. Abe, Superconductivity of $\mathrm{Ca}\left(\mathrm{Al}_{0.5} \mathrm{Si}_{0.5}\right)_{2}$, a ternary silicide with the $\mathrm{AlB}_{2}$-type structure, Appl. Phys. Lett. 80, 1019 (2002).

[23] M. Imai, E. El-Hadi Sadki, H. Abe, K. Nishida, T. Kimura, T. Sato, K. Hirata, and H. Kitazawa, Superconducting properties of single-crystalline $\mathrm{Ca}\left(\mathrm{Al}_{0.5} \mathrm{Si}_{0.5}\right) 2$ : A ternary silicide with the $\mathrm{AlB}_{2}$-type structure, Phys. Rev. B 68, 064512 (2003).

[24] B. Lorenz, J. Lenzi, J. Cmaidalka, R. L. Meng, Y. Y. Sun, Y. Y. Xue, and C. W. Chu, Superconductivity in the C32 intermetallic compounds $\mathrm{AAl}_{2-x} \mathrm{Si}_{\mathrm{x}}$, with $\mathrm{A}=\mathrm{Ca}$ and $\mathrm{Sr}$; and $0.6<x<1.2$, Physica C 383, 191 (2002).

[25] B. Lorenz, J. Cmaidalka, R. L. Meng, and C. W. Chu, Thermodynamic properties and pressure effect on the superconductivity in CaAlSi and SrAlSi, Phys. Rev. B 68, 014512 (2003).

[26] T. Nakagawa, M. Tokunaga, and T. Tamegai, Comparison of physical properties in BaAlSi and CaAlSi, Sci. Technol. Adv. Mater. 7, S108 (2006).

[27] S. Yamanaka, T. Otsuki, T. Ide, H. Fukuoka, R. Kumashiro, T. Rachi, K. Tanigaki, F. Guo, and K. Kobayashi, Missing superconductivity in BaAlSi with the $\mathrm{AlB}_{2}$ type structure, Physica C 451, 19 (2007).

[28] R. L. Meng, B. Lorenz, J. Cmaidalka, Y. S. Wang, Y. Y. Sun, J. Lenzi, J. K. Meen, Y. Y. Xue, and C. W. Chu, Study of intermetallic compounds isostructural to $\mathrm{MgB}_{2}$, IEEE Trans. Appl. Supercond. 13, 3042 (2003).

[29] R. Ma, G. Huang, W. Wang, H. Shu, S. Liu, and M. Liu, Effect of structure on the superconductivity of CaAlSi and SrAlSi: Density functional calculations, Physica C 468, 2233 (2008).

[30] M. J. Evans, Y. Wu, V. F. Kranak, N. Newman, A. Reller, F. J. Garcia-Garcia, and U. Häussermann, Structural properties and superconductivity in the ternary intermetallic compounds MAB $(\mathrm{M}=\mathrm{Ca}, \mathrm{Sr}, \mathrm{Ba} ; \mathrm{A}=\mathrm{Al}, \mathrm{Ga}, \mathrm{In} ; \mathrm{B}=\mathrm{Si}, \mathrm{Ge}, \mathrm{Sn})$, Phys. Rev. B 80, 064514 (2009).

[31] Whenever the chemical formula CaAlSi is used throughout this paper, then we always talk about the $6 \mathrm{H}$ polymorph, since this is the thermodynamic stable stoichiometric form, if not otherwise explicitly noted.

[32] S. Kuroiwa, H. Sagayama, T. Kakiuchi, H. Sawa, Y. Noda, and J. Akimitsu, Correlation between crystal structures of CaAlSi with and without superlattice and superconducting properties, Phys. Rev. B 74, 014517 (2006).

[33] H. Sagayama, Y. Wakabayashi, H. Sawa, T. Kamiyama, A Hoshikawa, S. Harjo, K. Uozato, A. K. Ghosh, M. Tokunaga, and T. Tamegai, Two types of multistack structures in $\mathrm{MgB}_{2}$ type superconductor CaAlSi, J. Phys. Soc. Jpn. 75, 043713 (2006).

[34] L. Boeri, J. S. Kim, M. Giantomassi, F. S. Razavi, S. Kuroiwa, J. Akimitsu, and R. K. Kremer, Pressure effects on the superconducting transition in nH-CaAlSi, Phys. Rev. B 77, 144502 (2008).

[35] S. Kuroiwa, K. Satoh, A. Koda, R. Kadono, K. Ohishi, W. Higemoto, and J. Akimitsu, Measurements on the vortex lattice of CaAlSi: Anisotropic response in magnetic penetration depth, J. Phys. Chem. Solids 68, 2124 (2007).

[36] B. Lorenz, J. Cmaidalka, R. L. Meng, Y. Y. Xue, and C. W. Chu, Thermodynamic and superconducting properties of the C32 intermetallic compounds CaAlSi and SrAlSi, Physica C 408-410, 171 (2004).

[37] S. Tsuda, T. Yokoya, S. Shin, M. Imai, and I. Hase, Identical superconducting gap on different Fermi surfaces of 
$\mathrm{Ca}\left(\mathrm{Al}_{0.5} \mathrm{Si}_{0.5}\right)_{2}$ with the $\mathrm{AlB}_{2}$ structure, Phys. Rev. B 69, 100506 (2004).

[38] J. Rodríguez-Carvajal, Recent advances in magnetic structure determination by neutron powder diffraction, Physica B 192, 55 (1993).

[39] R. C. Clark and J. S. Reid, The analytical calculation of absorption in multifaceted crystals, Acta Crystallogr. Sect. A 51, 887 (1995).

[40] O. V. Dolomanov, L. J. Bourhis, R. J. Gildea, J. A. Howard, and H. Puschmann, OLEX2: A complete structure solution, refinement and analysis program, J. Appl. Crystallogr. 42, 339 (2009).

[41] G. M. Sheldrick, SHELXT - Integrated space-group and crystal-structure determination, Acta Crystallogr. Sect. A 71, 3 (2015).

[42] G. M. Sheldrick, Crystal structure refinement with SHELXL, Acta Crystallogr. Sect. C 71, 3 (2015).

[43] R. Khasanov, Z. Guguchia, A. Maisuradze, D. Andreica, M. Elender, A. Raselli, Z. Shermadini, T. Goko, F. Knecht, E. Morenzoni et al., High pressure research using muons at the Paul Scherrer Institute, High Press. Res. 36, 140 (2016).

[44] A. Maisuradze, A. Shengelaya, A. Amato, E. Pomjakushina, and $\mathrm{H}$. Keller, Muon spin rotation investigation of the pressure effect on the magnetic penetration depth in $\mathrm{YBa}_{2} \mathrm{Cu}_{3} \mathrm{O}_{\mathrm{x}}$, Phys. Rev. B 84, 184523 (2011).

[45] Z. Guguchia, A. Amato, J. Kang, H. Luetkens, P. K. Biswas, G. Prando, F. von Rohr, Z. Bukowski, A. Shengelaya, H. Keller et al., Direct evidence for a pressure-induced nodal superconducting gap in the $\mathrm{Ba}_{0.65} \mathrm{Rb}_{0.35} \mathrm{Fe} 2 \mathrm{As} 2$ superconductor, Nat. Commun. 6, 8863 (2015).

[46] A. Suter and B. Wojek, Musrfit: A free platform-independent framework for $\mu$ SR data analysis, Phys. Procedia 30, 69 (2012).

[47] See Supplemental Material at http://link.aps.org/supplemental/ 10.1103/PhysRevResearch.3.033192 for details of the singlecrystal refinement for samples with $\mathrm{x}=0.2,0.4,0.5$, and 1 , as well as for the observed changes of the critical temperature after subsequent annealing and reheating of the samples.

[48] W. L. McMillan, Transition temperature of strong-coupled superconductors, Phys. Rev. 167, 331 (1968).

[49] F. von Rohr, H. Luo, N. Ni, M. Wörle, and R. J. Cava, Superconductivity and correlated Fermi liquid behavior in noncentrosymmetric $\mathrm{Ca}_{3} \mathrm{Ir}_{4} \mathrm{Ge}_{4}$, Phys. Rev. B 89, 224504 (2014).

[50] J. E. Sonier, J. H. Brewer, and R. F. Kiefl, $\mu$ SR studies of the vortex state in type-II superconductors, Rev. Mod. Phys. 72, 769 (2000).

[51] E. H. Brandt, Flux distribution and penetration depth measured by muon spin rotation in high- $T_{c}$ superconductors, Phys. Rev. B 37, 2349 (1988).

[52] S. Blundell, Spin-polarized muons in condensed matter physics, Contemp. Phys. 40, 175 (1999).

[53] R. Khasanov, A. Shengelaya, A. Maisuradze, F. L. Mattina, A. Bussmann-Holder, H. Keller, and K. A. Müller, Experimental Evidence for Two Gaps in the High-Temperature $\mathrm{La}_{1.83} \mathrm{Sr}_{0.17} \mathrm{CuO} 4$ Superconductor, Phys. Rev. Lett. 98, 057007 (2007).

[54] E. H. Brandt, Properties of the ideal Ginzburg-Landau vortex lattice, Phys. Rev. B 68, 054506 (2003).
[55] M. Tinkham, Introduction to Superconductivity (Krieger Publishing Company, Malabar, FIL, 1975).

[56] A. Carrington and F. Manzano, Magnetic penetration depth of $\mathrm{MgB}_{2}$, Physica C 385, 205 (2003).

[57] C. Niedermayer, C. Bernhard, T. Holden, R. K. Kremer, and K. Ahn, Muon spin relaxation study of the magnetic penetration depth in $\mathrm{MgB}_{2}$, Phys. Rev. B 65, 094512 (2002).

[58] R. Khasanov, M. Bendele, A. Amato, K. Conder, H. Keller, H.-H. Klauss, H. Luetkens, and E. Pomjakushina, Evolution of Two-Gap Behavior of the Superconductor $\mathrm{FeSe}_{1-x}$, Phys. Rev. Lett. 104, 087004 (2010).

[59] C. Niedermayer, C. Bernhard, U. Binninger, H. Glückler, J. L. Tallon, E. J. Ansaldo, and J. I. Budnick, Muon Spin Rotation Study of the Correlation between $T_{c}$ and $n_{s} / m^{\star}$ in Overdoped $\mathrm{Tl}_{2} \mathrm{Ba}_{2} \mathrm{CuO}_{6}+\delta$, Phys. Rev. Lett. 71, 1764 (1993).

[60] Y. Uemura, G. Luke, B. Sternlieb, J. Brewer, J. Carolan, W. Hardy, R. Kadono, J. Kempton, R. Kiefl, S. Kreitzman, P. Mulhern, T. M. Riseman, D. Ll. Williams, B. X. Yang, S. Uchida, H. Takagi, J. Gopalakrishnan, A. W. Sleight, M. A. Subramanian, C. L. Chien et al., Universal Correlations between $T_{c}$ and $n_{s} / m^{\star}$ (Carrier Density Over Effective Mass) in High- $T_{c}$ Cuprate Superconductors, Phys. Rev. Lett. 62, 2317 (1989).

[61] H. Kim, G. Preosti, and P. Muzikar, Penetration depth and impurity scattering in unconventional superconductors: $T=0$ results, Phys. Rev. B 49, 3544 (1994).

[62] V. G. Kogan, R. Prozorov, and V. Mishra, London penetration depth and pair breaking, Phys. Rev. B 88, 224508 (2013).

[63] L. Muechler, Z. Guguchia, J. C. Orain, J. Nuss, L. M. Schoop, R. Thomale, and F. O. von Rohr, Superconducting order parameter of the nodal-line semimetal NaAlSi, APL Mater. 7, 121103 (2019).

[64] F. O. von Rohr, J.-C. Orain, R. Khasanov, C. Witteveen, Z. Shermadini, A. Nikitin, J. Chang, A. R. Wieteska, A. N. Pasupathy, M. Z. Hasan, A. Amato et al., Unconventional scaling of the superfluid density with the critical temperature in transition metal dichalcogenides, Sci. Adv. 5, eaav8465 (2019).

[65] Z. Guguchia, F. von Rohr, Z. Shermadini, A. T. Lee, S. Banerjee, A. R. Wieteska, C. A. Marianetti, B. A. Frandsen, H. Luetkens, Z. Gong et al., Signatures of the topological $i+-$ superconducting order parameter in the type-II Weyl semimetal $\mathrm{T}_{\mathrm{d}}-\mathrm{MoTe}_{2}$, Nat. Commun. 8, 1082 (2017).

[66] R. Prozorov and R. W. Giannetta, Magnetic penetration depth in unconventional superconductors, Supercond. Sci. Tech. 19, R41 (2006).

[67] R. Khasanov, H. Luetkens, A. Amato, H.-H. Klauss, Z.-A. Ren, J. Yang, W. Lu, and Z.-X. Zhao, Muon spin rotation studies of $\mathrm{SmFeAsO}_{0.85}$ and $\mathrm{NdFeAsO}_{0.85}$ superconductors, Phys. Rev. B 78, 092506 (2008).

[68] F. von Rohr, A. Schilling, R. Nesper, C. Baines, and M. Bendele, Conventional superconductivity and charge-densitywave ordering in $\mathrm{Ba}_{1-\mathrm{x}} \mathrm{Na}_{\mathrm{x}} \mathrm{Ti}_{2} \mathrm{Sb}_{2} \mathrm{O}$, Phys. Rev. B 88, 140501(R) (2013).

[69] Z. Guguchia, D. J. Gawryluk, M. Brzezinska, S. S. Tsirkin, R. Khasanov, E. Pomjakushina, F. O. von Rohr, J. A. Verezhak, M. Z. Hasan, T. Neupert et al., Nodeless superconductivity and its evolution with pressure in the layered dirac semimetal $2 \mathrm{M}$ $\mathrm{WS}_{2}$, npj Quantum Mater. 4, 1 (2019). 